\title{
Über Congruenzen in Bezug auf einen Primzahlmodul.
}

Von Leopold Gegenbauer in Wien.

Sind

$$
z_{0}, z_{1}, \ldots, z_{r-1} \quad(r \leq p-1)
$$

$r$ nach dem Primzahlmodul $p$ unter einander und zu Null incongruente ganze Zahlen, so lassen sich, wie immer die ganzen Zahlen $a_{0}, a_{1}, \ldots, a_{r-1}$ beschaffen sein mögen, stets $r$ ganze Zahlen $\alpha_{0}, \alpha_{1}, \ldots, \alpha_{r-1}$, so bestimmen, dass die Congruenzen

$$
a_{k} \equiv \sum_{\lambda=0}^{\lambda=r-1} \alpha_{\lambda} z_{\lambda}^{k}(\bmod . p) \quad(k=0,1, \ldots, r-1)
$$

bestehen, weil die Determinante

$$
\left|z_{\lambda}^{k}\right|_{(k, \lambda=0,1, \ldots, r-1)}
$$

zu $p$ theilerfremd ist.

Bilden die Zablen $a_{k}(k=0,1, \ldots, p-2)$ ein Lösungssystem des Systemes linearer homogener Congruenzen

$$
\sum_{k=0}^{k=p-2} b_{k+\varrho} y_{k} \equiv 0(\bmod \cdot p)\left(\rho=0,1, \ldots, p-2 ; b_{h+p-1} \equiv b_{h}(\bmod \cdot p)\right) \text {, }
$$

so ergeben sich unter Benützung der Relationen (1) und auf Grund des Fermat'schen Satzes folgende zwei Congruenzensysteme

$$
\begin{aligned}
& \sum_{\lambda, \lambda=0}^{k, \lambda} \alpha_{\lambda} b_{k+e} z_{\lambda}^{k}+\sum_{k=r}^{k=1} b_{k+e} a_{k} \equiv 0(\bmod \cdot p)(\rho=0,1, \ldots p-2) \\
& \sum_{\lambda=0}^{\lambda=-2} a_{\lambda} z_{\lambda}^{p-1-e}\left(\sum_{k=0}^{k=1-2} b_{k} z_{\lambda}^{k}\right) \equiv 0(\bmod \cdot p)(\rho=0,1, \ldots, p-2),
\end{aligned}
$$

in deren zweiten die Marke am Summenzeichen anzeigt, dass nur jene Werte von $\lambda$ zu nehmen sind, für welche $\alpha_{\lambda}$ nicht durch $p$ theilbar ist.

Genügen die Zahlen

der Congruenz

$$
z_{0}, z_{1}, \ldots, z_{r-1}
$$

$$
\sum_{k=0}^{k=p-2} b_{k} x^{k} \equiv 0(\bmod , p)
$$


und demnach auch allen Congruenzen

$$
\sum_{k=0}^{k=p-2} b_{k+\varrho} x^{k} \equiv 0(\bmod \cdot p)(p=0,1, \ldots, p-2)
$$

so kann man das erste System auch in folgender Gestalt schreiben

$$
\sum_{k=r}^{k=p-2} b_{k+\varrho}\left\{a_{k}-\alpha_{0} z_{0}^{k}-\alpha_{1} z_{1}^{k}-\ldots-\alpha_{r-1} z_{r-1}^{k}\right\} \equiv 0(\bmod . p)(p=0,1, \ldots, p-2),
$$

in welcher es unmittelbar zu folgendem Satze führt:

I. Das System linearer homogener Congruenzen

$$
\sum_{k=0}^{k=p-2} b_{k+\varrho} y_{k}=0(\bmod \cdot p) \quad(\rho=0,1, \ldots, p-2)
$$

besitzt nur Lösungssysteme von der Form

$$
\sum_{\lambda=0}^{\lambda=0} \alpha_{\lambda} z_{\lambda}^{k}(k=0,1, \ldots, p-2)
$$

oder nicht, je nachdem es unter sämmtlichen in der Matrix

$$
\left\|b_{k+\varrho}\right\|_{(k=r, r+1, \ldots, p-2 ; \varrho=0,1, \ldots, p-2)}
$$

enthaltenen Determinanten $(p-r-1)^{\text {ter }}$ Ordnung zu $p$ theilerfremde gibt, oder nicht.

Das zweite der oben angegebenen Congruenzensysteme zeigt, dass das System linearer homogener Congruenzen

$$
\sum_{\lambda=r}^{\lambda=p-2} \alpha_{\lambda} z_{\lambda}^{p-l-\varrho} u_{\lambda} \equiv 0(\bmod . p) \quad(\rho=0,1, \ldots, p-2)
$$

ein Lösungssystem besitzt, dessen sämmtliche Elemente zu $p$ theilerfremd sind, falls die Zahlen $z_{0}, z_{1}, \ldots, z_{r-1}$ alle unter einander und von Null verschiedenen Wurzeln der Congruenz (2) sind. Daraus folgt aber sofort, dass alle in der Matrix

$$
\left\|\alpha_{2} z_{\lambda}^{p-1-\varrho}\right\|_{(i=r, r+1, \ldots, p-2 ; \varrho=0,1, \ldots, p-2)}
$$

enthaltenen Determinanten der Ordnung $p-r-1$ den Factor $p$ besitzen. Nun ist aber schon die erste von ihnen $(p=0,1, \ldots p-r-2)$ das Product ans einer zu $p$ theilerfremden Determinante und den in den eben benützten Congruenzen auftretenden Zahlen $\alpha_{\lambda}$, und daher muss jede Grösse $\alpha_{\hat{\imath}}$, deren Index $r-1$ übersteigt, durch $p$ theilbar sein. Man hat daher den Satz: 
II. Besitzt die Congruenz

$$
\sum_{k=0}^{k=p-2} b_{k} x^{k} \equiv 0(\bmod \cdot p)
$$

genan $r$ unter einander und von Null verschiedene Wurzeln $z_{0}, z_{1}, \ldots, z_{r-1}$, so lässt sich jedes Lösungssystem des Systemes linearer homogener Congruenzen

$$
\sum_{k=0}^{k=p-2} b_{k+\varrho} y_{k} \equiv 0(\bmod . p) \quad(\rho=0,1, \ldots, p-2)
$$

in der Form

darstellen.

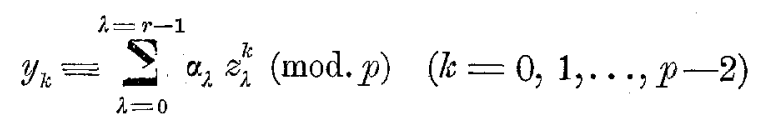

Dass dieser Satz auch umgekehrt gilt, ist unmittelbar ersichtlich.

Die Verbindung der zwei abgeleiteten Sätze liefert das folgende bemerkenswerte Theorem des Herrn Kronecker:

Die Summe aus der Anzahl der Wurzeln der Congruenz

$$
\sum_{k=0}^{k=p-2} b_{k} x^{k} \equiv 0(\bmod \cdot p)
$$

und dem Rangeihres Coefficientensystemes ist gleich $p-1$.

Der Satz II kann auch in der folgenden eleganten Fassung: ausgesprochen werden:

Das System ron linearen homogenen Congruenzen

$$
\sum_{k=0}^{k=p-2} b_{k+\varrho} y_{k} \equiv 0(\bmod \cdot p) \quad(\rho=0,1, \ldots, p-2)
$$

hat ebensoviele linear nnabhängige Lösungssysteme, als die Congruenz

$$
\sum_{k=0}^{k=p-2} b_{k} x^{k} \equiv 0(\bmod \cdot p)
$$

unter einander und von Null verschiedene Wurzeln besitzt.

In dieser von Herrn Kronecker auf andere Weise abgeleiteten Form erweist er sich als das zahlentheoretische Analogon des folgenden lïngst bekannten Theorems:

Bestehen zwischen $n$ Gröben $r$ lineare homogene Relationen, welche bei allen cyklischen Permutationen der Coefficienten erhalten bleiben, so gibt es für dieselben auch $r$ lineare homogene Relationen, deren Coëfficienten Einheitswurzeln sind. 